\title{
Correlação entre o índice morfológico das coroas dos incisivos inferiores e a estabilidade da correção do apinhamento ântero-inferior*
}

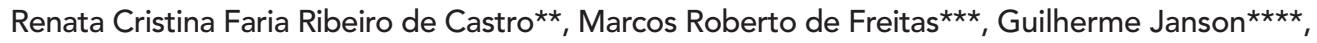 \\ Karina Maria Salvatore de Freitas ${ }^{\star \star \star \star \star}$
}

Resumo

Objetivo: investigar até que ponto as dimensões mesiodistais e vestibulolinguais das coroas dos incisivos inferiores contribuem para a estabilidade da correção do apinhamento ânteroinferior. Metodologia: foram avaliados 56 pacientes leucodermas, de ambos os gêneros (27 feminino e 29 masculino), que inicialmente apresentavam má oclusão de Classe I ou de Classe II, divisão 1 (28 cada), tratados com extrações dos quatro primeiros pré-molares e com mecânica Edgewise. Nenhum caso foi submetido a desgastes interproximais durante ou após o tratamento. As medidas foram realizadas nos modelos de estudo obtidos de cada caso nas fases pré, pós-tratamento e pós-contenção, totalizando na avaliação de 168 modelos inferiores. A idade média pré-tratamento foi de 13,23 anos, o tempo de tratamento de 2,11 anos e póstratamento foi de 5,12 anos. $\mathrm{O}$ índice de irregularidade de Little foi utilizado para quantificar o apinhamento ântero-inferior e o índice de Peck e Peck para mensuração das dimensões mesiodistais e vestibulolinguais dos mesmos. O teste de correlação de Pearson foi utilizado para determinar a significância de correlação entre a morfologia das coroas dos incisivos inferiores e a estabilidade. Secundariamente, investigou-se a presença de dimorfismo entre os gêneros e a existência de diferença entre os dois tipos de má oclusão inicial, em relação à estabilidade da correção do apinhamento ântero-inferior, utilizando o teste $t$ independente. Resultados e Conclusões: concluiu-se que a morfologia da coroa dos incisivos inferiores apresentou uma fraca correlação com a estabilidade pós-contenção. Nem o gênero dos pacientes, nem o tipo de má oclusão inicial influenciaram na estabilidade da correção do apinhamento ântero-inferior, cinco anos pós-tratamento.

Palavras-chave: Morfologia da coroa. Apinhamento ântero-inferior. Extração dentária. Ortodontia corretiva. Estabilidade.

* Baseado na Tese de Mestrado de Renata Cristina Faria Ribeiro de Castro, apresentada à Faculdade de Odontologia de Bauru-USP.

** Mestre e Doutoranda em Ortodontia pela Faculdade de Odontologia de Bauru da Universidade de São Paulo-USP.

** Professor Titular da Disciplina de Ortodontia da Faculdade de Odontologia de Bauru da Universidade de São Paulo-USP. Coordenador do Curso de Pós-graduação ao nível de Doutorado e Orientador da pesquisa.

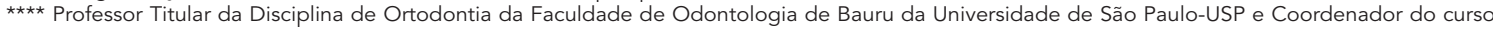
de Pós-graduação ao nível de Mestrado.

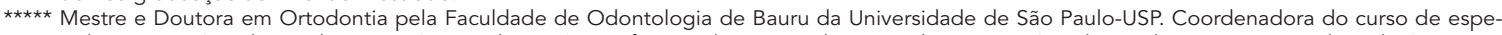
cialização em Ortodontia da Uningá (regional Bauru) e professora dos cursos de especialização em Ortodontia da Associação Paulista do CirurgiãoDentista (regional Bauru) e do Centro Universitário do Norte Paulista. 


\section{INTRODUÇÃO}

A chave do sucesso da Ortodontia é o exame dos pacientes tratados e a avaliação cuidadosa dos resultados. Os ortodontistas devem estar dispostos a examinar seus resultados criticamente, procurando sempre, por meio de uma média dos sucessos e falhas do tratamento, aprender e melhorar. A estabilidade em longo prazo das correções alcançadas durante o tratamento ortodôntico é um dos objetivos mais importantes e de maior dificuldade de obtenção na Ortodontia ${ }^{23}$. Pelo fato dos pacientes tenderem a priorizar, quase exclusivamente, o alinhamento dos seus incisivos e caninos e o apinhamento ântero-inferior ser a mais comum e reconhecida característica da má oclusão, os pacientes e os profissionais estão, indiscutivelmente, conscientes disto e ávidos pela sua correção ${ }^{21}$. No momento, não existe um consenso sobre a capacidade do ortodontista de, rotineiramente, obter estabilidade satisfatória em longo prazo. Alguma instabilidade pós-tratamento dos incisivos inferiores geralmente é inevitável, bem como o apinhamento associado com a idade. A raridade com que se encontram oclusões ideais anos após o término do tratamento ortodôntico e a similaridade das alterações pós-tratamento com as observadas em estudos longitudinais de oclusões normais ${ }^{7,45}$ sugerem que estas alterações fazem parte do processo normal de maturação da oclusão. A menos que a especialidade ortodôntica deseje aceitar a crença de que apenas poucos pacientes terão resultados satisfatórios muitos anos pós-contenção, estudos adicionais devem ser bem-vindos. Um dos tópicos que tem sido examinado com o passar dos anos e ainda em controvérsia na literatura é a associação de dentes mais largos com o apinhamento.

\section{Índices para avaliação quantitativa dos incisivos inferiores}

A utilização de índices para este tipo de avaliação deve garantir interpretação, facilidade de comunicação e aplicação uniforme dos critérios a serem utilizados. Termos como irregularidade dentária, sobreposição e apinhamento são subjetivos, não quantitativos, permitindo assim diferentes interpretações ${ }^{21}$. Atribuir um método quantitativo de medição entre um resultado ortodôntico que parece estável e um resultado que não pareça tão estável poderia ser útil em vários aspectos, servindo como dispositivo de auto-ensino para os ortodontistas.

Com o objetivo de verificar se os incisivos inferiores naturalmente bem alinhados possuíam características dimensionais distintas, Peck e Peck ${ }^{34}$ selecionaram dois grupos de mulheres americanas leucodermas, de ascendência européia, não tratadas ortodonticamente, com idade variando de 17 a 27 anos - sendo um com todos os dentes permanentes irrompidos até os segundos molares, ausência de tratamento ortodôntico prévio, contato proximal presente e ausência de sobreposição dos incisivos inferiores, e o segundo grupo foi escolhido sem critério. Em ambos os grupos, os diâmetros mesiodistal e vestibulolingual máximo de cada incisivo inferior foram medidos diretamente na boca. O diâmetro mesiodistal máximo foi encontrado na, ou próximo à, borda incisal; entretanto, o diâmetro vestibulolingual máximo, na maior parte dos casos, está abaixo da margem gengival, sendo então necessário posicionar a ponta do compasso subgengivalmente, impedindo assim a utilização de modelos de estudo. Concluíram ${ }^{34}$ que a forma dentária (dimensões mesiodistal e vestibulolingual - MD/ VL) é um fator determinante na presença ou ausência do apinhamento dos incisivos inferiores. A partir desse dado, para apresentar a base científica e aplicação clínica de um novo método para detecção e avaliação dos desvios da forma dentária dos incisivos inferiores, Peck e Peck ${ }^{32}$ propuseram um índice para Ortodontia clínica, utilizando a seguinte fórmula:

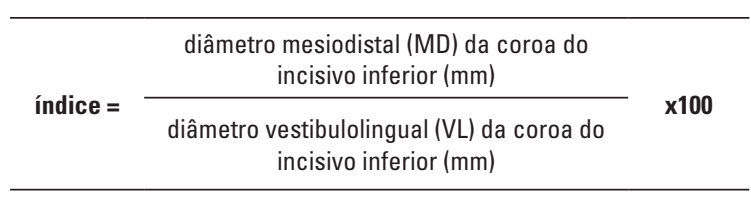


A fórmula foi aplicada à mesma amostra utilizada no estudo anterior ${ }^{34} \mathrm{e}$ os dados foram analisados estatisticamente. Esse índice nada mais é que uma expressão numérica da forma da coroa. Os autores $^{32}$ concluíram que os ortodontistas devem considerar a forma dentária da coroa dos incisivos inferiores e o índice MD/VL para o manejo ortodôntico bem sucedido do tratamento das irregularidades dos incisivos inferiores.

Em virtude da possibilidade das posições dos dentes ântero-inferiores serem um fator limitante no tratamento e estabilidade, seria lógico desenvolver um índice de diagnóstico que refletisse precisamente a posição desses dentes. Três anos depois, Little ${ }^{21}$ propôs um método quantitativo para avaliar as irregularidades dos dentes ânteroinferiores, envolvendo as medições da distância linear real dos pontos de contato anatômicos de cada incisivo inferior até o ponto de contato anatômico do dente adjacente, onde as somas destas cinco irregularidades representam a distância para o qual os pontos de contato devem ser movidos para atingir o alinhamento (Fig. 4). Este índice é classificado de acordo com os seguintes critérios:

- 0 = alinhamento perfeito;

- 1 - 3 = apinhamento mínimo;

- 4 - 6 = apinhamento moderado;

- 7 - 9 = apinhamento severo;

- 10 = apinhamento muito severo.

Este índice ${ }^{21}$ não tem uma avaliação do comprimento do arco e não leva em conta a análise cefalométrica, análise facial, idade, morfologia dentária, efeito de hábitos, entre outros. Por outro lado, oferece um guia para quantificar o apinhamento ântero-inferior, auxiliando muitos ortodontistas em suas pesquisas, pois além de determinar as condições pré-tratamento também avalia as possíveis mudanças pós-tratamento, além de ser útil nos programas de saúde pública e assistenciais.

\section{Morfologia da coroa dos incisivos e o apinha- mento ântero-inferior}

Embora exista uma discreta tendência de inci- sivos mais estreitos estarem associados com melhor alinhamento, em algumas ocasiões larguras mesiodistais mais estreitas não podem assegurar uma estabilidade em longo prazo, em casos tratados ortodonticamente ${ }^{14}$. Apesar da forma dos incisivos inferiores ser definida por Peck e Peck ${ }^{32}$ como um fator importante no apinhamento ântero-inferior, a questão da sua aplicabilidade foi mais amplamente discutida por Smith, Davidson e $\mathrm{Gipe}^{47}$, que encontraram que as dimensões MD/ VL e o índice de irregularidade de Little apresentavam correlação com o apinhamento. No entanto, ressaltaram que a largura mesiodistal isolada teve maior correlação com o apinhamento do que com as médias de proporções de forma, definidas por Peck e Peck ${ }^{32}$, concluindo que o uso das medições de tamanho dentário como um guia para procedimentos clínicos é uma simplificação de um problema muito complexo. Howe, McNamara e O'Connor ${ }^{18}$ fizeram uma investigação para determinar até que ponto o tamanho dos dentes e o tamanho do arco isoladamente contribuem para o apinhamento dentário. Independente se o tamanho dentário foi comparado individualmente ou se a comparação se deu com a soma mesiodistal de todo arco, não ocorreu diferença significativa no tamanho dentário entre os grupos com e sem apinhamento. Com o propósito de verificar se as dimensões mesiodistais e vestibulolinguais dos incisivos inferiores podem prever uma maior estabilidade das correções, Gilmore e Little ${ }^{14}$ examinaram 164 casos do Departamento de Ortodontia da Universidade de Washington, a maior parte tratada com a técnica Edgewise e os demais com extração de quatro pré-molares sem tratamento subseqüente. Todos os casos apresentavam Classe I ou II de Angle no pré-tratamento e todos possuíam bom alinhamento dos incisivos no final do tratamento ativo. Após avaliarem as dimensões dos incisivos inferiores e a sua relação com o apinhamento, concluíram que, para se prever a estabilidade pós-contenção, nenhuma correlação clinicamente útil foi encontrada entre o índice de Peck 
e Peck ${ }^{32}$ e outras variáveis dos modelos de estudo e cefalométricas, concluindo que larguras mesiodistais menores para os incisivos inferiores não garantiram uma melhora na estabilidade em longo prazo $^{14}$. Puneky, Sadowsky e Begole ${ }^{35}$, no mesmo ano, investigaram a contribuição das dimensões mesiodistal (MD) e vestibulolingual (VL) das coroas dos incisivos inferiores e suas proporções de forma (MD/VL x 100) em um período médio de 20 anos pós-contenção. Uma amostra de adultos com más oclusões não tratadas foi acrescentada para a obtenção de maiores informações sobre a estabilidade do grupo tratado. $\mathrm{O}$ alinhamento dos incisivos foi avaliado pelo índice de irregularidade de Little ${ }^{21}$ e todas as medidas foram registradas com compasso modificado, de precisão de $0,1 \mathrm{~mm}$. $\mathrm{O}$ resultado deste estudo ${ }^{35}$ mostrou que a forma e o tamanho dos incisivos inferiores não contribuem significantemente para o alinhamento dos incisivos após o tratamento ortodôntico ativo.

Para estabelecer se há correlação entre a forma da coroa dos incisivos e o apinhamento, Rhee e Nahm ${ }^{36}$ pesquisaram uma amostra, dividindo-a em dois grupos de acordo com o índice de irregularidade de $L_{i t l l e}{ }^{21}$, sendo um com apinhamento (índice de irregularidade maior que 3) e outro normal (índice de irregularidade menor ou igual a 3). Observaram que a média do valor mesiodistal para o grupo com apinhamento era significantemente maior na área incisal e menor na área cervical; concluindo que a prevalência de apinhamento é maior em indivíduos com forma triangular dos incisivos inferiores. Todavia, os autores $^{36}$ acrescentaram que a avaliação da forma da coroa dos incisivos como fator etiológico do apinhamento, prevenção da recidiva e estabilidade após o tratamento ortodôntico não pode ser feita apenas com estes resultados e que estudos adicionais devem ser realizados para determinação da correlação da proporção da largura, estabilidade e recidiva. Um novo método (digital) para medição dos diâmetros das coroas dos incisivos inferiores e sua relação com o apinhamento foi proposto por Shah, Elcock e Brook ${ }^{41}$. Para tanto, avaliaram os modelos de estudo de indivíduos leucodermas não tratados ortodonticamente com graus variados de apinhamento. Não foi encontrada diferença significativa entre a discrepância dente-arco dos lados direito e esquerdo, e não puderam ser estabelecidos previsores clínicos do apinhamento dos incisivos inferiores pela forma das coroas destes dentes.

Segundo a revisão de literatura, observa-se que poucos autores ${ }^{32,36,47}$ demonstraram correlações entre a largura dentária e o apinhamento; além disso, as diferenças na metodologia aplicada e nas amostras estudadas levaram alguns autores ${ }^{36,47}$ a comentários relevantes sobre a estrutura dentária; ou seja, que esta tem apenas um pequeno papel na causa do apinhamento tardio no arco inferior. Enquanto outros autores ${ }^{14,35,41}$ encontraram correlações não significantes, evidenciando em seus estudos que a morfologia da coroa dos incisivos inferiores não exerce influência na estabilidade ou recidiva da correção do apinhamento ânteroinferior.

\section{Apinhamento ântero-inferior e o desenvolvi- mento da oclusão}

Os resultados de estudos longitudinais começam a esclarecer as alterações pós-tratamento ativo (pós-contenção) presente em casos tratados ortodonticamente. O problema era a incapacidade em determinar se estas alterações ocorrem principalmente como resultado da terapia ortodôntica ou se fazem parte do desenvolvimento maturacional ${ }^{45}$. A terapia ortodôntica pode alterar o curso destas contínuas alterações fisiológicas, por algum tempo, e possivelmente até mesmo revertê-las; no entanto, após a mecanoterapia e o período de contenção, o desenvolvimento do processo maturacional prossegue ${ }^{16}$. Van Der Linden ${ }^{49}$ descreveu a expressão "apinhamento terciário", que se refere ao apinhamento que ocorre durante o período da adolescência e pós-adolescência. Os resultados mostraram que os incisivos inferiores e, em menor 
extensão, os superiores, se verticalizam e podem apinhar na época em que o crescimento da maxila já cessou e particularmente o da mandíbula continua. Arcos dentários idealmente alinhados podem começar a mostrar uma sobreposição dos dentes anteriores, um fenômeno que é observado mais no gênero masculino que no feminino. Isso está relacionado às diferenças entre os dois gêneros no crescimento facial tardio. A presença dos terceiros molares parece ter pouco efeito nesse tipo de apinhamento, já que ele também acontece quando não há terceiros molares. Para o autor ${ }^{49}$, a continuação do crescimento mandibular, numa época que há pouco ou nenhum crescimento do resto do complexo craniofacial, parece ser o fator contribuinte mais importante para a ocorrência desse apinhamento. Sinclair e Little ${ }^{45}$ avaliaram modelos dentários de pacientes com oclusões normais não tratadas, a fim de descrever a natureza e extensão das mudanças vistas durante o desenvolvimento da oclusão da dentadura mista (9-10 anos), início da dentadura permanente (12-13 anos) e dentadura permanente adulta (19-20 anos). O índice de irregularidade dos incisivos se manteve estável da dentadura mista até a dentadura permanente jovem, seguidos por um pequeno, mas estatisticamente significante, aumento dos 13 aos 20 anos. Quando comparado com os estudos pós-contenção de casos tratados, o apinhamento dos incisivos inferiores apresentou um índice de irregularidade maior, comparado ao grupo normal; ou seja, o apinhamento dos incisivos inferiores no grupo tratado aumentou duas vezes mais rápido que no grupo normal, no período pós-contenção. Estas diferenças deveriam sugerir que, embora a natureza das alterações (aumento do apinhamento) seja similar, a extensão destas alterações é diferente nos indivíduos tratados e não tratados. Lembrando que a idade média nos casos tratados tinha uma variação de 10 anos a mais do que a do grupo não tratado. Talvez, com o tempo o grupo não tratado também apinhará na mesma extensão ${ }^{27,42}$. Considerando que o apinhamento pós-tratamento e sua variação entre adultos têm sido uma preocupação contínua dos ortodontistas, em relação à estabilidade, Buschang e Shulman ${ }^{7}$ buscaram informações quanto à prevalência desse problema. A amostra foi composta por indivíduos sem tratamento ortodôntico prévio. Os resultados demonstraram que quase metade da população apresentou pouco ou nenhum apinhamento; ou seja, 23\% têm irregularidade moderada e $17 \%$ têm problemas severos, para os quais os tratamentos devem ser considerados altamente necessários. Os terceiros molares irrompidos não são associados com o aumento do apinhamento. Apesar das diferenças terem sido pequenas, os homens demonstraram irregularidade dos incisivos significantemente maior do que as mulheres; os melanodermas demonstraram menos irregularidade dos incisivos do que os leucodermas e os americanos mexicanos. A irregularidade aumentou de forma curvilínea com a idade, com maiores aumentos ocorridos durante ou no final da adolescência e início da terceira década de idade. Finalmente, apesar das diferenças individuais no apinhamento terem sido estudadas e explicadas, os determinantes primários permanecem não identificados.

Como demonstrado nesta revisão, mais estudos são necessários para compreensão de um dos principais objetivos do tratamento ortodôntico: a estabilidade. Observou-se que existem dúvidas no que diz respeito à morfologia das coroas dos incisivos inferiores e à recidiva da correção do apinhamento ântero-inferior, independente da quantidade de apinhamento antes do tratamento e da quantidade de alteração durante o tratamento. Diante de tal contexto, a fim de oferecer uma perspectiva clínica, este estudo tem por objetivo investigar principalmente as dimensões mesiodistais e vestibulolinguais das coroas dos incisivos inferiores e secundariamente avaliar a presença de dimorfismo entre os gêneros e a diferença existente entre dois tipos de má oclusão inicial, com relação à estabilidade da correção do apinhamento ântero-inferior. 


\section{MATERIAL E MÉTODOS Material}

A amostra foi selecionada de 570 documentações ortodônticas completas, tratados no Curso de Pós-Graduação em Ortodontia, da Universidade de São Paulo - Faculdade de Odontologia de Bauru.

Os critérios básicos de seleção foram que os indivíduos deveriam apresentar, no pré-tratamento, todos os dentes permanentes irrompidos (com exceção dos $3^{\text {os }}$ molares), más oclusões de Classe I e Classe II, divisão 1 (de meia Classe II até Classe II completa), tratamento ortodôntico corretivo com extração dos primeiros pré-molares superiores e inferiores, ausência de desgastes interproximais no arco inferior durante ou após o tratamento, bom alinhamento dos incisivos no final do tratamento e documentação ortodôntica realizada por um período mínimo de 5,12 anos pós-tratamento. Como critérios adicionais, incluíram-se: distribuição equilibrada entre os gêneros e entre as idades (em anos) dos pacientes no pré-tratamento. $\mathrm{O}$ índice de irregularidade de Little pré-tratamento variou de alinhamento perfeito a severo. A amostra constituiu-se de 56 pacientes, leucodermas, de ambos os gêneros (27 gênero feminino e 29 masculino), que inicialmente ao tratamento ortodôntico apresentavam más oclusões de Classe I e II divisão 1 (28 cada). Os dados referentes à idade média inicial, final de tratamento e fase pós-tratamento se

Tabela 1 - Dados referentes às idades (em anos) ao início, ao final do tratamento e pós-tratamento.

\begin{tabular}{cccccc}
\hline idade (em anos) & média & d.p. & máximo & mínimo & n \\
\hline inicial & 13,23 & 1,28 & 15,69 & 10,44 & 56 \\
final & 15,33 & 1,51 & 18,75 & 12,27 & 56 \\
pós-tratamento & 20,45 & 1,59 & 23,44 & 15,74 & 56 \\
\hline
\end{tabular}

encontram na tabela 1 e os dados relacionados aos tempos de tratamento, contenção e pós-tratamento encontram-se na tabela 2.

Todos os modelos foram obtidos de moldagens efetuadas com alginato e vazados em gesso-pedra, com o auxílio de um vibrador. Utilizaram-se também placas de mordida confeccionadas com cera rosa $\mathrm{n}^{\circ} 7$ para articular os modelos em intercuspidação, para posterior recorte e acabamento, segundo a técnica de Almeida, Pinzan e Santos ${ }^{1}$.

\section{Método de obtenção das medidas}

Objetivando melhor padronização durante as medições mesiodistais das coroas dos dentes superiores e inferiores, Yamaguto ${ }^{50}$ propôs, conjuntamente com um profissional técnico devidamente credenciado pela empresa Norberto Mischi (Norberto Mischi Soluções Técnicas Ltda. EPP, São Paulo/SP), modificar as pontas ativas de um paquímetro digital original (Fig. 1), marca Mitutoyo, com capacidade de $150 \mathrm{~mm}$, resolução.

\section{Variáveis estudadas}

1) Índice de irregularidade de Little ${ }^{21}$ : somatória $(\mathrm{A}+\mathrm{B}+\mathrm{C}+\mathrm{D}+\mathrm{E})$ do deslocamento dos cinco

Tabela 2 - Dados referentes aos tempos (em anos) do tratamento, contenção, pós-tratamento e tempo total de avaliação.

\begin{tabular}{|c|c|c|c|c|c|}
\hline $\begin{array}{c}\text { tempo } \\
\text { (em anos) }\end{array}$ & média & d.p. & máximo & mínimo & n \\
\hline tratamento & 2,11 & 0,64 & 4,00 & 0,82 & 56 \\
\hline contenção & 1,63 & 1,19 & 4,0 & 0,0 & 56 \\
\hline $\begin{array}{l}\text { pós-trata- } \\
\text { mento }\end{array}$ & 5,12 & 0,86 & 7,54 & 2,06 & 56 \\
\hline $\begin{array}{c}\text { tempo total } \\
\text { de avaliação }\end{array}$ & 7,22 & 0,92 & 9,07 & 5,06 & 56 \\
\hline
\end{tabular}

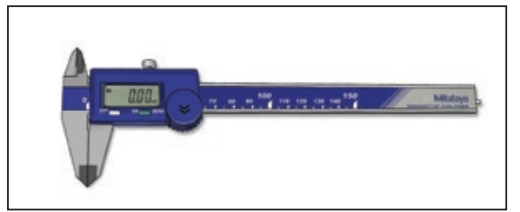

FIGURA 1 - Paquímetro digital Mitutoyo com pontas ativas originais.

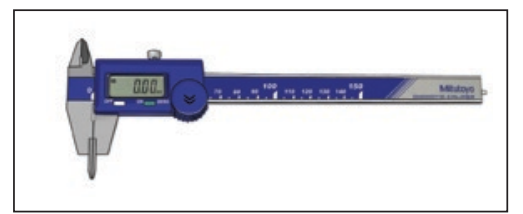

FIGURA 2 - Paquímetro digital Mitutoyo com pontas ativas modificadas.

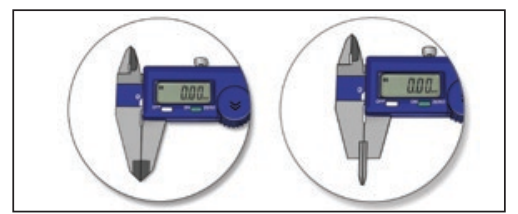

FIGURA 3 - Visão aproximada das pontas ativas do paquímetro digital Mitutoyo original e modificado. 
pontos de contato anatômico dos dentes ânteroinferiores (Fig. 4). A medição foi realizada com um paquímetro digital modificado, com precisão de $0,01 \mathrm{~mm}$, posicionado paralelamente ao plano oclusal (Fig. 5).

Foi efetuada uma modificação da face de medição, substituindo as pontas ativas de um paquímetro digital original, implantando duas placas de aço de $3 \mathrm{~mm}$ de largura e $2 \mathrm{~mm}$ de espessura, com as extremidades (pontas ativas) biseladas e rebaixadas na parte da face de medição, a fim de realizar os procedimentos de medição e obtenção de valores mais precisos (Fig. 2). Para que o aparelho continuasse com a mesma fidelidade de mensuração, foi realizada e devidamente testada a aferição do mesmo, pela empresa Norberto Mischi (Fig. 2, 3).

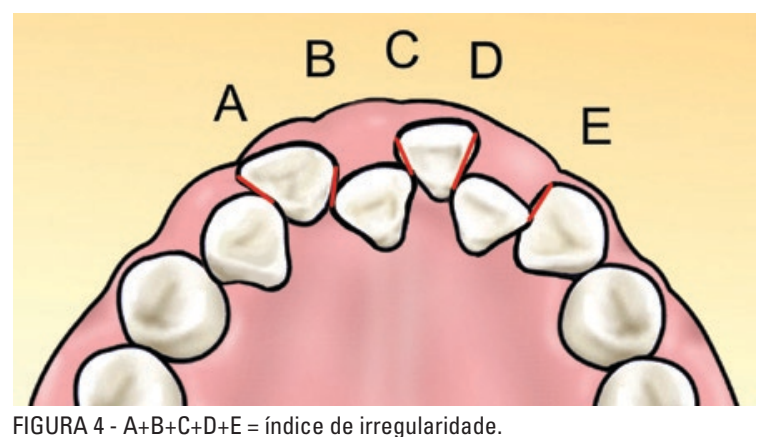

FIGURA $4-\mathrm{A}+\mathrm{B}+\mathrm{C}+\mathrm{D}+\mathrm{E}=$ índice de irregularidade

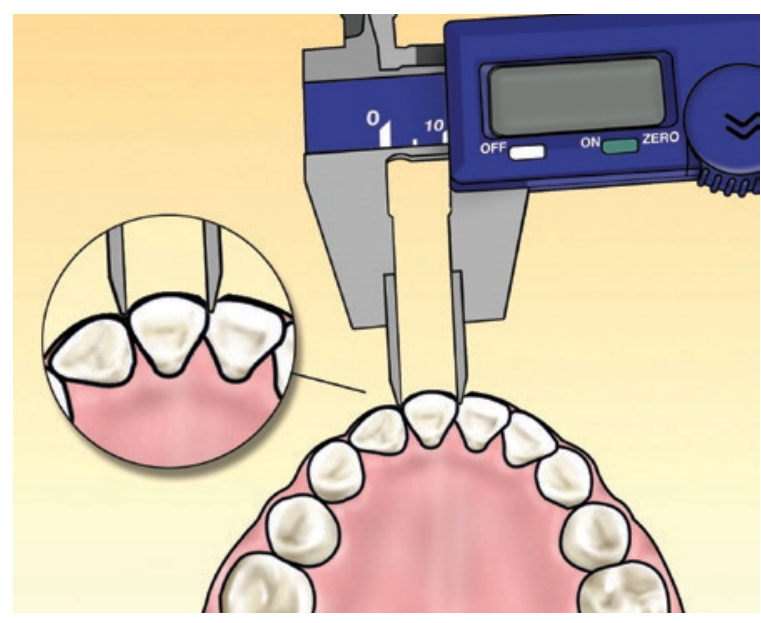

FIGURA 6 - Diâmetro mesiodistal da coroa do incisivo inferior - paquímetro paralelo ao plano oclusal e paralelo ao solo (fundo amarelo).
Após avaliação criteriosa e com o objetivo de alcançar melhor forma de mensuração das medidas mesiodistal e vestibulolingual das coroas dos incisivos inferiores, e devido à ausência no mercado de um paquímetro digital com pontas ativas específicas para realização das medições referidas, decidiu-se utilizar, neste estudo, o mesmo paquímetro digital proposto no estudo de Yamaguto ${ }^{50}$.

2) Índice Peck e Peck ${ }^{32}$ : diâmetros mesiodistal e vestibulolingual da coroa dos incisivos inferiores, medidos o mais perpendicularmente possível com relação ao longo eixo do dente e no maior diâmetro da coroa de cada incisivo inferior (Fig. 6, 7).

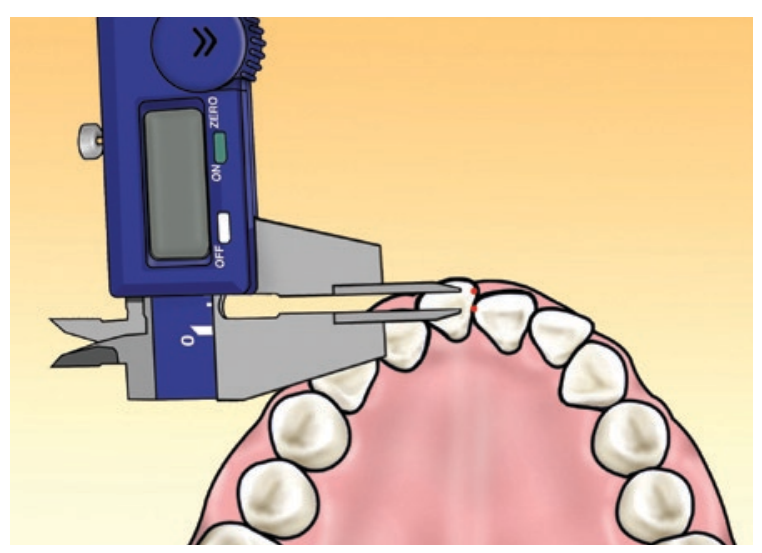

FIGURA 5 - Técnica de medição do índice de irregularidade - o paquímetro deve ser colocado paralelo ao plano oclusal, medindo apenas o apinhamento linear horizontal dos pontos de contatos anatômicos.

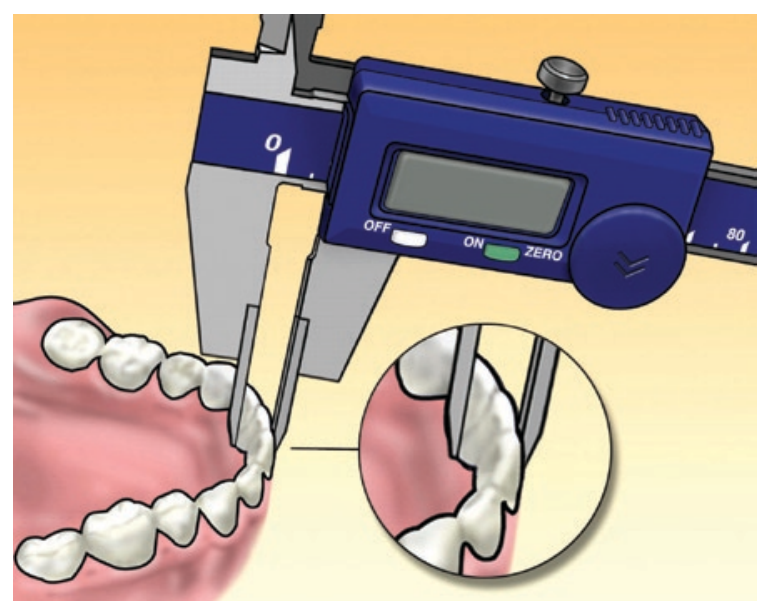

FIGURA 7 - Diâmetro vestibulolingual da coroa do incisivo inferior - paquímetro perpendicular à borda incisal da coroa do incisivo (seguindo o longo eixo da coroa clínica dos incisivos inferiores). 


\section{Análise estatística}

Todas as análises estatísticas foram desenvolvidas pelo programa computadorizado Statistica (Statistica 6.0 ${ }^{\mathrm{TM}}$, Statistical Software for Windows Version 6.0). Com o intuito de determinar a confiabilidade da pesquisa, foram medidos novamente os modelos de estudo de 28 pacientes, aleatoriamente selecionados e remedidos pelo mesmo examinador, com um mês de intervalo entre as medições ${ }^{17}$. Determinou-se a diferença entre a primeira e segunda mensuração de cada modelo e aplicou-se a fórmula de Dahlberg ${ }^{8}$ para o cálculo da magnitude dos erros casuais. O erro sistemático foi detectado comparando-se as medições por meio do teste $t$ dependente ${ }^{17}$. Para determinar a significância da correlação entre a morfologia da coroa dos incisivos inferiores em relação à estabilidade da correção do apinhamento ântero-inferior utilizou-se o teste de correlação de Pearson.

\section{RESULTADOS}

Os resultados mostram o tratamento estatístico dos dados, em forma de tabelas, sendo considerados estatisticamente significantes para $\mathrm{p}<0,05$.

\section{Erro do método (Tab. 3)}

De forma geral, os erros casuais neste trabalho foram bastante reduzidos para o índice de irregularidade de Little e para os incisivos centrais

Tabela 3 - Avaliação do erro do método intra-examinador. Diferença entre a primeira e segunda medição (milímetros ou percentagem), erro casual e erro sistemático.

\begin{tabular}{|c|c|c|c|c|c|c|}
\hline \multirow{2}{*}{ medidas } & \multicolumn{2}{|c|}{$1^{\text {a }}$ medição } & \multicolumn{2}{|c|}{ 2ª medição } & \multirow{2}{*}{$\begin{array}{c}\text { erro } \\
\text { casual } \\
\text { Dahlberg }\end{array}$} & \multirow{2}{*}{$\begin{array}{c}\text { erro } \\
\text { sistemático } \\
\mathbf{p}\end{array}$} \\
\hline & média & d.p. & média & d.p. & & \\
\hline $\begin{array}{l}\text { índice de } \\
\text { Little (mm) }\end{array}$ & 2,37 & 2,90 & 2,44 & 2,95 & 0,20 & 0,22 \\
\hline $32(\%)$ & 98,03 & 6,47 & 97,19 & 7,65 & 3,43 & 0,36 \\
\hline $31(\%)$ & 91,32 & 5,45 & 92,22 & 5,61 & 3,72 & 0,37 \\
\hline $41(\%)$ & 91,43 & 5,48 & 92,08 & 5,59 & 3,71 & 0,52 \\
\hline $42(\%)$ & 98,39 & 6,82 & 98,43 & 7,7 & 3,58 & 0,97 \\
\hline
\end{tabular}

e laterais inferiores, direito e esquerdo. Levando em consideração os altos valores das variáveis estudadas em porcentagem para os incisivos inferiores, o erro casual foi considerado extremamente pequeno e aceitável. O maior significado dos erros casuais refere-se ao seu poder de aumentar o desvio padrão das médias obtidas ${ }^{8}$. Como os erros casuais para as variáveis em estudo foram pequenos, concluiu-se que os desvios-padrão encontrados realmente reflitam a variabilidade das medidas em questão. Nenhuma variável apresentou erro sistemático para $\mathrm{p}<0,05$. Os erros sistemáticos se manifestam quando um determinado parâmetro é persistentemente sub ou superestimado, podendo ocorrer quando um pesquisador modifica a sua técnica de mensuração após certo tempo, ou quando tende subconscientemente a direcionar os resultados, de acordo com as suas expectativas com relação às conclusões do estudo ${ }^{17}$. Desta forma, a ausência de erros sistemáticos e os valores reduzidos para o erro casual permitiram a realização desta pesquisa, transmitindo confiabilidade no momento das medições, quanto ao paquímetro selecionado para execução destas (Tab. 3).

\section{Má oclusão (Tab. 4)}

A tabela 4 evidencia que a má oclusão inicial não exerce um fator de influência em relação à estabilidade da correção do apinhamento ânteroinferior no período pós-contenção.

Tabela 4 - Resultado do teste t independente, usado para avaliar a existência de diferença entre os dois tipos de má oclusão inicial com relação à estabilidade da correção do apinhamento ântero-inferior.

\begin{tabular}{cccccc}
\hline & \multicolumn{2}{c}{ Classe I } & \multicolumn{2}{c}{ Classe II } & \\
\cline { 2 - 5 } & \multicolumn{2}{c}{ (n=28) } & \multicolumn{2}{c}{ (n=28) } \\
\hline variável & média & d.p. & média & d.p. & p \\
\hline Little inicial (T1) & 4,70 & 2,96 & 5,48 & 3,61 & 0,38 \\
Little (T3-T2) & 1,06 & 1,15 & 0,95 & 1,17 & 0,71 \\
\hline
\end{tabular}




\section{Dimorfismo entre gêneros (Tab. 5)}

Os resultados da tabela 5 reportam que a presença de dimorfismo entre gêneros não exerce nenhum papel importante em relação à estabilidade no período pós-contenção.

\section{Estatística descritiva (Tab. 6, 7)}

A tabela 6 apresenta a estatística descritiva das médias e os desvios-padrão dos dentes $32,31,41$ e 42.

\section{Correlação entre o índice morfológico das co- roas dos incisivos inferiores e estabilidade pós- tratamento (Tab. 8)}

Os resultados mostram que os diâmetros (MD/VL) das coroas dos incisivos inferiores não contribuem significantemente (o valor de $\mathrm{p}$ variou de 0,34 a 0,93 ) para o alinhamento destes dentes no período médio de 5 anos pós-tratamento.

\section{DISCUSSÃO}

Os objetivos específicos do tratamento devem ser pensados com a estabilidade em mente, mas

Tabela 5 - Resultado do teste t independente para verificar a presença de dimorfismo entre gêneros com relação à estabilidade da correção do apinhamento ântero-inferior.

\begin{tabular}{|c|c|c|c|c|c|}
\hline \multirow{2}{*}{ variável } & \multicolumn{2}{|c|}{ feminino ( $n=27)$} & \multicolumn{2}{|c|}{ masculino $(n=29)$} & \multirow{2}{*}{$\mathbf{p}$} \\
\hline & média & d.p. & média & d.p. & \\
\hline Little (T3-T2) & 0,98 & 1,02 & 1,02 & 1,28 & 0,91 \\
\hline Little Inicial (T1) & 4,85 & 3,7 & 5,31 & 2,93 & 0,60 \\
\hline
\end{tabular}

Tabela 7 - Médias e desvios-padrão do índice de Little inicial, final, pós-tratamento e a diferença entre o índice de Little pós-contenção e final de tratamento (T3-T2).

\begin{tabular}{cccccc}
\hline Little & média & d.p. & máximo & mínimo & $\mathbf{n}$ \\
\hline inicial (T1) & 5,09 & 3,29 & 14,01 & 0 & 56 \\
final (T2) & 0,05 & 0,20 & 1,00 & 0 & 56 \\
pós-contenção (T3) & 1,05 & 1,20 & 4,19 & 0 & 56 \\
T3 -T2 (pós - final) & 1,00 & 1,15 & 4,06 & 0 & 56 \\
\hline
\end{tabular}

esta não pode ser absoluta. Os ortodontistas devem procurar conseguir para seus pacientes uma estabilidade aceitável, visto que as alterações das relações dentárias, anos após a finalização da fase ativa do tratamento, são esperadas, mas não necessariamente indicam recidiva ${ }^{38}$. Está nos olhos de cada um decidir se um caso recidivou ou se a alteração pós-tratamento ainda apresenta-se aceitável $^{38}$. Independentemente da etiologia desta recidiva, a irregularidade dos incisivos inferiores é geralmente a precursora da recidiva do apinhamento superior, da sobremordida e da deterioração do tratamento ${ }^{21,22}$. Por outro lado, se o profissional acredita que os melhores esforços são desnecessários, a qualidade da finalização ortodôntica certamente seria comprometida e se a qualidade do tratamento for importante na estabilidade, esta deve ser uma preocupação importante, pois é o fator mais passível de controle do ortodontista. A falta de conhecimento sobre todos os fatores que levam à estabilidade não deve impedir a qualidade do tratamento como um dos objetivos principais, onde o conceito de uma estabilidade aceitável deve ser compreendido apenas como o reconhecimento de

Tabela 6 - Médias e desvios-padrão dos dentes 32, 31, 41 e 42 no início do tratamento.

\begin{tabular}{cccccc}
\hline dente & média & d.p. & máximo & mínimo & n \\
\hline 32 & 98,2 & 6,3 & 115,1 & 84,4 & 56 \\
31 & 91,6 & 5,6 & 103,8 & 78,1 & 56 \\
41 & 91,6 & 5,7 & 103,8 & 78,1 & 56 \\
42 & 98,1 & 6,3 & 115,1 & 83,1 & 56 \\
\hline
\end{tabular}

Tabela 8 - Resultado do teste de correlação de Pearson entre os valores do índice de Peck e Peck ${ }^{32}$ obtidos neste trabalho e estabilidade da correção do apinhamento ântero-inferior.

\begin{tabular}{cccc}
\hline dente & $\mathbf{r}$ & $\mathbf{p}$ & $\mathbf{n}$ \\
\hline 32 & $-0,06$ & 0,68 & 56 \\
31 & 0,13 & 0,34 & 56 \\
41 & 0,11 & 0,41 & 56 \\
42 & $-0,01$ & 0,93 & 56 \\
\hline
\end{tabular}


limitações biológicas e não como justificativa para casos clínicos mal finalizados.

Alguns estudos que avaliaram a correlação entre a forma da coroa dos incisivos e o apinhamento não levaram em consideração o diâmetro vestibulolingual ${ }^{18,36,41}$, como proposto por Peck e Peck $^{32}$, e nem se propuseram a avaliar a mesma amostra em longo prazo $32,33,36,47$. A Odontologia atual é baseada em evidências científicas, portanto, a avaliação de amostras no período pós-contenção oferece maior credibilidade às pesquisas, a fim de identificar as razões dos efeitos deletérios do apinhamento pós-tratamento ativo $6,10,14,20,22,25,27,31,35,38$.

\section{Má oclusão inicial}

O presente estudo encontrou que a má oclusão inicial não exerce um fator de influência sobre a recidiva da correção do apinhamento ântero-inferior. Outros estudos ${ }^{9,14,27,37,38,44}$ que avaliaram o apinhamento no período pós-contenção assemelhamse a este quanto à seleção da má oclusão inicial de Classe I e II, entretanto, poucos levaram em consideração a avaliação da relação entre a má oclusão inicial com o apinhamento pós-contenção ${ }^{14,37}$.

Little, Wallen e Riedel ${ }^{27}$; Shields, Little e Cha$\mathrm{pko}^{44}$, dentre outras variáveis estudadas, verificaram também a relação da má oclusão inicial com relação à recidiva do apinhamento ântero-inferior e concluíram que esta variável não exerce influência sobre o apinhamento dos incisivos inferiores na fase pós-contenção, corroborando desta forma o presente estudo.

\section{Dimorfismo entre gêneros}

O presente estudo avaliou a presença de dimorfismo entre gêneros com relação à recidiva, não encontrando nenhuma correlação. Apesar de alguns autores $^{12,13}$ terem encontrado presença de dimorfismo entre os gêneros, estes confirmaram que o maior dimorfismo encontrado entre gêneros ocorre nos diâmetros mesiodistais dos primeiros e segundos molares inferiores, seguidos pelos caninos superiores e inferiores. Resultados de outros estudos ${ }^{4,15}$ também foram semelhantes. Os resultados da pesquisa conduzida por Lyssel e Myrberg ${ }^{28}$ encontraram que os dentes que exibiram maior grau de variação no diâmetro mesiodistal foram: o incisivo lateral superior (em média 8,5\%) e o primeiro molar superior (em média 4,6\%), na dentadura permanente. Este estudo assemelha-se com os resultados encontrados por Hattab, Al-Khateeb e Sultan ${ }^{15}$, que também verificaram que em ambos os gêneros os incisivos laterais superiores apresentaram maior variabilidade, com o coeficiente de variação de $8,8 \%$, e o primeiro molar a menor variabilidade, com o coeficiente de variação de $5,8 \%$. Neste estudo ${ }^{15}$, os caninos apresentaram o maior dimorfismo entre os gêneros, em relação ao tamanho da coroa dentária. Bishara et al. ${ }^{4}$ mostraram evidências de dimorfismo entre os gêneros, principalmente em caninos e molares, sendo mais largos no gênero masculino, no entanto, os incisivos não se apresentaram diferentes. Baum e Cohen $^{3}$, ao compararem o seu estudo com a mesma amostra do estudo de Garn, Lewis e Kerewsky ${ }^{11}$, não encontraram diferença entre as larguras mesiodistais dos pacientes de ambos os gêneros, resultado oposto ao obtido pelos autores ${ }^{11}$, onde os pacientes do gênero masculino apresentaram dentes maiores que os pacientes do gênero feminino. Todavia, os estes $^{3}$ efetuaram mensurações mesiodistais das coroas dentárias em um maior número de mulheres do que de homens.

\section{Idade}

O apinhamento ântero-inferior pode receber influência do crescimento tardio da mandíbula ${ }^{25,37,49}$ e, desta forma, aumentar com a idade $23,45,46$. Por este motivo, para melhor padronização da amostra, foram selecionados casos com as idades dos pacientes (em anos) compativeis em todos os períodos avaliados, nos estágios pré-tratamento, pós-tratamento e, principalmente, na fase pós-tratamento (Tab. 1).

\section{Desgastes interproximais}

Outro critério utilizado foi a ausência de desgastes interproximais nos incisivos e caninos infe- 
riores durante ou após o tratamento. Como o objetivo deste estudo foi avaliar a real influência da morfologia das coroas dos incisivos inferiores, com relação à recidiva da correção do apinhamento ântero-inferior, não seria lógico avaliar casos onde tivessem sido realizados desgastes interproximais, pois estes alteram de forma irreversível a forma das coroas dos dentes ${ }^{43}$. Freitas et al. ${ }^{9}$, ao avaliarem pacientes tratados sem extrações, encontraram uma porcentagem média de recidiva relativamente pequena. Ao avaliar os casos que sofreram desgastes interproximais, não foi observada diferença significante entre os casos tratados com e sem desgastes interproximais, em relação à recidiva. Gilmore e Little $^{14}$ relataram que a redução da largura dos incisivos inferiores não pode ser esperada como algo que vá produzir estabilidade do alinhamento póscontenção, mesmo porque casos tratados com extrações no arco inferior também não apresentaram estabilidade em longo prazo $^{24,25}$.

\section{Contenção}

Todos os pacientes da amostra utilizaram contenção no arco inferior colada de canino a canino (3x3), por 1 a 2 anos (média 1,63 anos). Alguns estudos $^{37,38,48,49}$ relataram que o final do crescimento ântero-posterior dos maxilares, particularmente o crescimento terminal da mandíbula, pode induzir a formação de apinhamento tardio ânteroinferior ou terciário; por este fato, a manutenção da contenção no arco inferior até o final desta fase de crescimento é uma indicação precisa. No entanto, outros pesquisadores ${ }^{23,24}$ indicam sua manutenção de forma permanente ou por um período de tempo indeterminado, pois o apinhamento ântero-inferior é um fenômeno contínuo, principalmente dos 30 aos 40 anos $^{23}$ e depois disto ${ }^{5,46}$. A transferência de responsabilidade pela estabilidade para o paciente, na forma de contenção pela vida toda, é a melhor opção, sem dúvida, para certos problemas, mas isso é prático ou necessário para o paciente? No presente estudo, o tempo de contenção foi relativamente curto, porém, a média de recidiva encontrada foi pequena $(1 \mathrm{~mm})$. Isto pode estar relacionado ao fato de alguns pacientes terem apresentado índice de irregularidade inicial com apinhamento mínimo? Little e Riedel ${ }^{24}$ avaliaram, por um período mínimo de 10 anos pós-contenção, pacientes que apresentavam espaços generalizados nos dentes anteriores, inicialmente ao tratamento ortodôntico, concluindo que o grau de constrição das dimensões do arco é imprevisível, pelo fato dos espaços não reabrirem em nenhum caso avaliado. Baseado neste estudo ${ }^{24}$, o índice de irregularidade mínimo ou alinhamento perfeito no início do tratamento parece não prever o resultado pós-contenção em longo prazo. Sendo assim, a contenção por tempo indeterminado seria necessária para aqueles pacientes que exigem do ortodontista a perfeição do alinhamento ântero-inferior em longo prazo. Sadowski et al. ${ }^{39}$ encontraram alinhamento relativamente bom dos incisivos inferiores na fase de pós-contenção (recidiva mínima) e creditaram este resultado ao tempo de contenção aumentado, em média de 8 anos. Salienta-se que o tempo de contenção do presente estudo não interferiu na estabilidade alcançada no período pós-contenção. Little, Wallen e Riedel ${ }^{27}$ consideraram que, em longo prazo, o comportamento do alinhamento ântero-inferior é variável e imprevisível e que o tempo de contenção não foi útil para estabelecer o prognóstico do resultado em longo prazo.

\section{Estudo em modelos}

Segundo Peck e Peck ${ }^{32}$, a melhor forma de medição dos diâmetros mesiodistais e vestibulolinguais das coroas dos incisivos inferiores seria a avaliação clínica direta de cada paciente. A base para essa afirmativa, segundo os autores ${ }^{32}$, se dá principalmente pela correta medição do diâmetro vestibulolingual máximo, que se encontra subgengivalmente na maior parte dos casos; logo, seria difícil a realização desta medida em modelos. Neste estudo, esse tipo de avaliação seria praticamente impossivel, por se tratar de um estudo retrospectivo. Portanto, as mensurações das medidas foram re- 
alizadas diretamente nos modelos de estudo, antes, após o tratamento e no pós-contenção, tendo, nos mesmos, os dentes representados adequadamente, como relatado nos estudos de Hunter e Priest ${ }^{19}$, os quais obtiveram mais segurança em medidas feitas sobre modelos do que diretamente na boca do paciente. Gilmore e $\mathrm{Littl}^{14}$, em um estudo piloto, compararam as medidas feitas em pacientes e em modelos de estudo e não encontraram diferenças. Por outro lado, é quase unânime na literatura ${ }^{36,47}$ que o diâmetro mesiodistal é relatado como possível fator primário para o apinhamento e não o diâmetro vestibulolingual. Além disso, medições subgengivais com um instrumental que não pudesse ser esterilizado levariam à quebra da cadeia asséptica, com risco de infecção cruzada para o paciente.

\section{Índice de irregularidade de Little}

$\mathrm{O}$ índice de irregularidade de Little ${ }^{21}$ foi escolhido para avaliação do apinhamento inferior nos modelos de estudo, por permitir uma avaliação em modelos de gesso, ser altamente reproduzível e confiável, e por ser o índice mais utilizado em artigos sobre estabilidade e/ou recidiva do apinhamento ântero-inferior encontrados na literatura ortodôntica ${ }^{9,10,14,35,36,47}$. Portanto, por meio deste índice, foi possível quantificar a severidade do apinhamento ântero-inferior previamente ao tratamento ortodôntico, ao final do tratamento e cinco anos pós-tratamento.

\section{Avaliação da recidiva do apinhamento ântero-inferior}

Recidiva, readaptação fisiológica, acomodação pós-contenção e outros termos que descrevem as alterações dos casos tratados ortodonticamente induzem boas e más lembranças aos ortodontistas. Os ortodontistas tendem a revelar e documentar aqueles casos que permanecem sem alterações significantes com o decorrer do tempo, como exemplos de seu sucesso e habilidade. Os outros casos, nos quais o objetivo ideal do tratamento não foi atingido, podem surpreender pela melhora ocorrida com o tempo. $\mathrm{Na}$ verdade, a recidiva pode ocorrer inesperadamente e sem aparente explicação. Os pacientes já estão cientes que merecem mais do que bons resultados oclusais e estéticos ao final do tratamento; eles naturalmente também almejam a manutenção destes resultados. Um dos mais importantes problemas ainda não resolvidos na Ortodontia é a estabilidade em longo prazo do alinhamento dos incisivos inferiores; apesar de muitos esforços já terem sido despendidos para resolver, minimizar ou compreender o problema, ainda não foi encontrada uma resposta definitiva. Alguma instabilidade pós-tratamento dos incisivos inferiores é inevitável, bem como o apinhamento associado com a idade. Mas quanto apinhamento pós-tratamento deve ser esperado: mínimo, moderado ou severo?

Os resultados mostraram que, neste estudo, o valor do índice de irregularidade pós-contenção foi considerado mínimo, segundo Little ${ }^{21}(1,05 \mathrm{~mm})$. Os pacientes apresentaram apinhamento ânteroinferior (índice de irregularidade de Little) prétratamento de, em média, 5,09mm, que foi corrigido para 0,05 ao final do tratamento corretivo, e no pós-contenção recidivou $1 \mathrm{~mm}$. Praticamente $80 \%$ da amostra estudada apresentaram alinhamento ântero-inferior clinicamente aceitável, segundo Little ${ }^{21}$. Estes resultados corroboraram o estudo de Boley et al. ${ }^{6}$, que demonstraram que o apinhamento medido pelo índice de irregularidade de Little diminuiu 5,3mm durante o tratamento e aumentou $0,7 \mathrm{~mm}$ durante o período pós-contenção. Dos pacientes avaliados, $80 \%$ apresentaram alinhamento satisfatório $(<3,5 \mathrm{~mm})$ dos incisivos inferiores no pós-contenção e nenhum estava na categoria severa $(>6, \mathrm{~mm})$. Os autores ${ }^{6}$ concluíram que podem ser obtidos resultados satisfatórios em longo prazo para a maior parte dos pacientes de Classe I com extrações de 4 pré-molares. A estabilidade pós-contenção encontrada neste estudo também foi sustentada por outros trabalhos realizados em amostras tratadas com extrações no 
arco inferior ${ }^{38,48}$. Para Sadowsky e Sakols ${ }^{38}$, enquanto no início do tratamento 35\% da amostra apresentaram apinhamento ântero-inferior acima da variação considerada normal $(3 \mathrm{~mm})$, somente $15 \%$ dos casos apresentam um grau de apinhamento acima de $3 \mathrm{~mm}$ no período médio de 12 anos pós-contenção, confirmando que a maioria das correções foram mantidas. Ao avaliar casos em 2 períodos de pós-contenção, Vaden, Harris e Gardner ${ }^{48}$ verificaram um decréscimo na recidiva do apinhamento ântero-inferior de $1,6 \mathrm{~mm}$ quando avaliados 6 anos pós-contenção e 2,6mm quando novamente avaliados num período médio de 15 anos pós-contenção. Os casos avaliados apresentaram índice de Little inicial de $4,7 \mathrm{~mm}$ e no pós-tratamento apresentaram valores próximos de zero. Os resultados otimistas do presente estudo e de outros $^{6,38,48}$ não são sustentados por alguns estudos clássicos da Universidade de Washington ${ }^{2,22,23}$, que relataram a dificuldade em manter o alinhamento ântero-inferior, independentemente da filosofia e técnica de tratamento, gênero e idade do paciente. Todavia, todos estes estudos foram avaliados por um período mínimo de 10 anos pós-contenção; portanto, a diferença destes resultados para a presente pesquisa pode residir no tempo de avaliação pós-contenção. Outro fator que poderia ser questionado quanto à estabilidade pós-contenção desta pesquisa seria o fato do índice de irregularidade no pré-tratamento apresentar-se menor (média de $5,09 \mathrm{~mm}$ ) do que nos estudos de Little, Wallen e Riedel $^{27}$ (média de 7,31 mm) e Little, Riedel e Ar$\operatorname{tun}^{25}$ (média de 7,41 mm). Concluíram que quanto maior a mudança induzida pelo tratamento, maior a tendência para mudanças no pós-tratamento ${ }^{27}$. Entretanto, os estudos de Little, Riedel e Engst ${ }^{26}$ (média de 4,13mm) e McReynolds e Little ${ }^{29}$ (média de 4,00mm) com índice de irregularidade inicial menores que os desta pesquisa, não apresentaram estabilidade em 10 anos pós-contenção. Com base nestes estudos ${ }^{26,29}$, depreendemos que a quantidade de apinhamento ântero-inferior inicial pode não ser garantia de estabilidade pós-contenção.

\section{Correlação da morfologia dos incisivos inferio- res com a recidiva}

Os resultados mostraram que os diâmetros MD/VL das coroas dos incisivos inferiores não contribuem significantemente com a recidiva ou a estabilidade pós-contenção; ou seja, apresentam uma fraca correlação ( $p$ variou de 0,34 a 0,93 ) para o alinhamento destes dentes no período médio de 5 anos pós-tratamento. Outros autores ${ }^{14,18,35}$ estão de acordo com estes resultados. Embora alguns estudos anteriores ${ }^{32,36,47}$ tenham observado uma relação entre os diâmetros dos incisivos inferiores com o apinhamento ou alinhamento desses dentes, nenhum deles teve acompanhamento em longo pra$\mathrm{zo}^{14}$. Além disso, a avaliação de amostras não tratadas ortodonticamente ${ }^{32,36,47}$ ignora os efeitos do tratamento ortodôntico, bem como a possibilidade de que os pacientes tratados possam representar em uma população como um todo ${ }^{14}$. Peck e Peck ${ }^{32}$ avaliaram amostras não tratadas ortodonticamente e encontraram uma associação entre a morfologia da coroa dos incisivos inferiores com o alinhamento destes dentes; por outro lado, os limites da estabilidade deste estudo ${ }^{32}$ não permitem que os resultados sejam passivamente confiáveis, pois se limitam em apenas um único período de avaliação, não confirmando com nenhum estudo adicional a eficácia da manutenção dos resultados em longo prazo $^{10,20}$.

A importância da medida subgengival dos incisivos inferiores e a estabilidade pós-contenção foi questionada por alguns autores $s^{30,36,41,47}$, que observaram que a simples medida do comprimento mesiodistal dos incisivos inferiores é mais útil que as proporções mesiodistais e vestibulolinguais destes dentes. Rhee e $\mathrm{Nahn}^{36}$ avaliaram modelos de estudo de indivíduos aleatoriamente selecionados e dividiram a amostra em 2 grupos, um com índice de irregularidade maior que $3 \mathrm{~mm}$ e outro grupo com irregularidade menor ou igual a $3 \mathrm{~mm}$. Nenhum paciente foi submetido a tratamento ortodôntico e este estudo não teve acompanhamento em longo prazo. Os resultados mostraram que a 
proporção da largura dos incisivos no grupo com apinhamento foram maiores do que aquelas com incisivos bem alinhados; ou seja, dentes com formato triangular são mais propensos à instabilidade. Desta forma, os autores ${ }^{36}$ concluíram que a largura mesiodistal dos incisivos é uma das características do apinhamento, solicitando atenção no diagnóstico e tratamento destes dentes triangulares, bem como para o protocolo de contenção; no entanto, sugeriram que estudos adicionais devem ser realizados para determinação da correlação da proporção da largura, estabilidade e recidiva do apinhamento ântero-inferior. Outros estudos concordam com a sugestão final deste trabalho ${ }^{36}$ e afirmam que larguras mesiodistais estreitas das coroas dos incisivos inferiores também não podem garantir estabilidade em longo prazo de casos ortodonticamente tratados ${ }^{14,18,35,40}$.

Shah, Elcock e Brook ${ }^{41}$ apresentaram uma metodologia de medição por imagens digitais para quantificar os diâmetros das coroas dos incisivos inferiores e assim verificar sua relação com o apinhamento. Os autores reconhecem que algum grau do apinhamento dos incisivos inferiores geralmente ocorre na população ortodôntica, pré e póstratamento em longo prazo e que a etiologia dessa condição é multifatorial. Este trabalho foi amplamente questionado por Peck $^{33}$, pois os autores ${ }^{41}$ não encontraram indicadores clínicos do apinhamento dos incisivos inferiores pela forma da coroa dos incisivos inferiores. Este método digital parece muito útil para medição do diâmetro mesiodistal das coroas dos incisivos inferiores, mas não poderia ser aplicado no presente estudo, pois seria inviável medir o diâmetro vestibulolingual por meio de imagens bidimensionais; seria necessária uma imagem tridimensional para medir o diâmetro mesiodistal e vestibulolingual. Mas se o maior diâmetro mesiodistal da coroa dos incisivos inferiores encontra-se na borda incisal, não se justifica desgastar o modelo até o nível do ponto médio mesial e distal para realizar esta medição. Desta forma, a medição com paquímetro seria mais fácil e prática; ou seja, compensa utilizar a técnica digital se esta facilitar o trabalho, mas se o erro do método foi aceitável não há motivos para abandonar o método com paquímetro digital.

Estudos longitudinais de oclusão normal demonstram que a movimentação dentária faz parte do processo normal de maturação da oclusão durante toda vida; portanto, o apinhamento dos incisivos poderá aumentar com a idade, mesmo em casos de oclusão normal ${ }^{5,7,45,46}$.

\section{CONSIDERAÇÕES FINAIS}

Poderia haver questionamentos quanto ao tempo de acompanhamento pós-contenção do presente estudo, sugerindo nova avaliação em um tempo maior de acompanhamento para estas mesmas modalidades de tratamento, mas não podemos esquecer que a variabilidade biológica ${ }^{5,7,45,46,49}$ pode confundir mesmo os estudos mais cuidadosos.

Conseguir bons resultados ao final do tratamento ortodôntico é possivel diante de um diagnóstico e plano de tratamento adequados. É razoável tentar, por meio de pesquisas científicas, solucionar algo que se almeja, mas que nem sempre é possível de ser alcançado. Aceitar passivamente a possibilidade da recidiva nos torna frustrados e ansiosos. A magia da ciência está justamente na tentativa de descobrir ou complementar algo que ainda não foi provado e que talvez, num futuro próximo, possa ser solucionado. A Ortodontia vem progredindo cientificamente e tecnologicamente, desde o último século, e muitos protocolos foram alterados para obtenção dos objetivos do tratamento, principalmente com relação à estabilidade em longo prazo do alinhamento dos incisivos inferiores.

Com relação às alterações suscitadas pelo tratamento com extrações dos quatro primeiros prémolares e de acordo com a proposição, metodologia e resultados deste estudo, pode-se inferir que não houve correlação entre a forma da coroa dos incisivos inferiores e a estabilidade da correção do apinhamento ântero-inferior cinco anos pós-tratamento. Nem o gênero dos pacientes, nem o tipo 
de má oclusão inicial influenciaram na estabilidade da correção do apinhamento ântero-inferior em cinco anos pós-tratamento. Indubitavelmente, há problemas com a crença de que a maior parte dos pacientes terá recidiva em extensão satisfatória; no entanto, este estudo oferece documentação adicional relacionada à estabilidade pós-contenção. o presente estudo e ao Prof. Dr. Renato Rodrigues de Almeida (Professor Associado da Disciplina de Ortodontia da Faculdade de Odontologia de Bauru-USP), pela amizade, senso crítico e disposição em colaborar com a seleção do melhor instrumental para mensuração das medidas obtidas nesta pesquisa.

\title{
AGRADECIMENTOS
}

Ao Prof. Osvaldo Tatsuo Yamaguto, por gentilmente ceder o paquímetro digital modificado para

\section{Correlation between the morphologic index of crowns of mandibular incisors and stability of correction of mandibular anterior crowding}

\begin{abstract}
Aim: This study investigated the extent to which the mesiodistal and buccolingual dimensions of the crowns of mandibular incisors contribute with the stability of correction of mandibular anterior crowding. Methods: For that purpose, 56 white patients of both genders (27 females and 29 males) were evaluated, who initially presented Class I or Class II division 1 malocclusion (28 patients each), treated with extraction of the four first premolars and edgewise mechanics. No interproximal stripping was performed during or after treatment. Measurements were performed on the dental casts achieved from each case at pretreatment, posttreatment and postretention, adding up to 168 mandibular dental casts. The mean age at pretreatment was 13.23 years, the mean treatment time was 2.11 years and the mean post-retention time was 5.12 years. The Little irregularity index was used to quantify the mandibular anterior crowding, and the Peck and Peck index was applied for measurement of the mesiodistal and buccolingual ratios of the crowns of mandibular incisors. The Pearson correlation test was applied to determine the significance of correlation between the morphology of the crowns of mandibular incisors and stability. Dimorphism between genders and the existence of difference between the two types of initial malocclusion were also investigated by the independent $t$ test. Results and Conclusion: Analysis of the results revealed that the morphology of crown of mandibular incisors was not significantly correlated with postretention stability. The gender and type of initial malocclusion also did not influence the stability of correction of mandibular anterior crowding at five years posttreatment.
\end{abstract}

Keywords: Crown morphology. Mandibular anterior crowding. Tooth extraction. Corrective orthodontics. Stability.

\section{REFERÊNCIAS}

1. ALMEIDA, R. R.; PINZAN, A.; SANTOS, E. C. A. Preparo de modelos ortodônticos. Rev. Fac. Odontol. Lins, Lins, v. 6, n. 1, p. 42-47, jan./jun. 1993.

2. ARTUN, J.; GAROL, J. D.; LITTLE, R. M. Long-term stability of mandibular incisors following successful treatment of Class II, Division 1, malocclusions. Angle Orthod., Appleton, v. 66, no. 3, p. 229-238, 1996.

3. BAUM, B. J.; COHEN, M. M. Decreased odontometric sex difference in individuals with dental agenesis. Am. J. Phys. Anthropol., New York, v. 38, no. 3, p. 739-741, May 1973

4. BISHARA, S. E. et al. Mesiodistal crown dimensions in Mexico and the United States. Angle Orthod., Appleton, v. 56, no. 4, p. 315-323, Oct. 1986.
5. BISHARA, S. E. et al. Changes in the maxillary and mandibular tooth size-arch length relationship from early adolescence to early adulthood: a longitudinal study. Am. J. Orthod. Dentofacial Orthop., St. Louis, v. 95, no. 1, p. 46-59, Jan. 1989.

6. BOLEY, J. C. et al. Long-term stability of Class I premolar extraction treatment. Am. J. Orthod. Dentofacial Orthop. St. Louis, v. 124, no. 3, p. 277-287, Sept. 2003.

7. BUSCHANG, P. H.; SHULMAN, J. D. Incisor crowding in untreated persons 15-50 years of age: United States, 1988-1994. Angle Orthod., Appleton, v. 73, no. 5, p. 502-508, Oct. 2003.

8. DAHLBERG, G. Statistical methods for medical and biological students. New York: Interscience, 1940.

9. FREITAS, K. M. et al. Postretention relapse of mandibular anterior crowding in patients treated without mandibular premolar extraction. Am. J. Orthod. Dentofacial Orthop., St. Louis, v. 125, no. 4, p. 480-487, Apr. 2004. 
10. FREITAS, M. R. et al. Correlation between mandibular incisor crown morphologic index and postretention stability. Am. J. Orthod. Dentofacial Orthop., St. Louis, v. 129, no. 4, p. 559561, 2006.

11. GARN, S. M.; LEWIS, A. B.; KEREWSKY, R. S. Size Interrelationships of the mesial and distal teeth. J. Dent. Res., Alexandria, v. 44, p. 350-354, Mar./Apr. 1965.

12. GARN, S. M.; LEWIS, A. B.; KEREWSKY, R. S. Communalities in the size differences of teeth of brothers and sisters. Arch. Oral Biol., Oxford, v. 12, no. 5, p. 575-581, May 1967

13. GARN, S. M. et al. Genetic control of sexual dimorphism in tooth size. J. Dent. Res., Alexandria, v. 46, no. 5, p. 963-972, Sept./Oct. 1967.

14. GILMORE, C. A.; LITTLE, R. M. Mandibular incisor dimensions and crowding. Am. J. Orthod., St. Louis, v. 86, no. 6, p. 493502, Dec. 1984

15. HATTAB, F. N.; AL-KHATEEB, S.; SULTAN, I. Mesiodistal crown diameters of permanent teeth in Jordanians. Arch. Oral Biol. Oxford, v. 41, no. 7, p. 641-645, July 1996.

16. HOROWITZ, S. L.; HIXON, E. H. Physiologic recovery following orthodontic treatment. Am. J. Orthod., St. Louis, v. 55, no. 1, p. 1-4, Jan. 1969.

17. HOUSTON, W. J. B. The analysis of erros in orthodontic measurements. Am. J. Orthod., St. Louis, v. 83, no. 5, p. 382-390, May 1983.

18. HOWE, R. P.; MCNAMARA, J. A., JR.; O'CONNOR, K. A. An examination of dental crowding and its relationship to tooth size and arch dimension. Am. J. Orthod., St. Louis, v. 83, no. 5 , p. 363-373, May 1983

19. HUNTER, W. S.; PRIEST, W. R. Errors and discrepancies in measurement of tooth size. J. Dent. Res., Alexandria, v. 39, no. 2, p. 405-414, Mar. 1960

20. JANSON, G.; FREITAS, K. M. S.; CASTRO, R. C. F. R. Authors response to Dr. Peck. Am. J. Orthod. Dentofacial Orthop. St. Louis, v. 131, no. 1, p. 3-4, 2007.

21. LITTLE, R. M. The irregularity index: a quantitative score of mandibular anterior alignment. Am. J. Orthod., St. Louis, v. 68 , no. 5 , p. 554-563, Nov. 1975

22. LITTLE, R. M. Stability and relapse of dental arch alignment. Br. J. Orthod., Oxford, v. 17, no. 3, p. 235-241, Aug. 1990

23. LITTLE, R. M. Stability and relapse of mandibular anterior alignment: University of Washington studies. Semin. Orthod., Philadelphia, v. 5, no. 3, p.191-204, Sept. 1999.

24. LITTLE, R. M.; RIEDEL, R. A. Postretention evaluation of stability and relapse - mandibular arches with generalized spacing Am. J. Orthod. Dentofacial Orthop., St. Louis, v. 95, no. 1, p. 37-41, Jan. 1989

25. LITTLE, R. M.; RIEDEL, R. A.; ARTUN, J. An evaluation of changes in mandibular anterior alignment from 10 to 20 years postretention. Am. J. Orthod. Dentofacial Orthop., St. Louis v. 93, no. 5, p. 423-428, May 1988.

26. LITTLE, R. M.; RIEDEL, R. A.; ENGST, E. D. Serial extraction of first premolars-postretention evaluation of stability and relapse. Angle Orthod., Appleton, v. 60, no. 4, p. 255-262, 1990.

27. LITTLE, R. M.; WALLEN, T. R.; RIEDEL, R. A. Stability and relapse of mandibular anterior alignment - first premolar extraction cases treated by traditional edgewise orthodontics. Am. J. Orthod., St. Louis, v. 80, no. 4, p. 349-365, Oct. 1981.

28. LYSELL, L.; MYRBERG, N. Mesiodistal tooth size in the deciduous and permanent dentitions. Eur. J. Orthod., Oxford, v. 4, no. 2, p.113-122, May 1982

29. MCREYNOLDS, D. C.; LITTLE, R. M. Mandibular second premolar extraction--postretention evaluation of stability and relapse. Angle Orthod., Appleton, v. 61, no. 2, p.133-44, 1991.

30. NORDERVAL, K.; WISTH, P. J.; BOE, O. E. Mandibular anterior crowding in relation to tooth size and craniofacial morphology. Scand. J. Dent. Res., Copenhagen, v. 83, no. 5, p. 267-273, Sept. 1975.

31. ORMISTON, J. P. et al. Retrospective analysis of long-term stable and unstable orthodontic treatment outcomes. Am. J. Orthod. Dentofacial Orthop., St. Louis, v. 128 , no. 5, p. 568$574,2005$.
32. PECK, H.: PECK, S. An index for assessing tooth shape deviations as applied to the mandibular incisors. Am. J. Orthod., St. Louis, v. 61, no. 4, p. 384-401, Apr. 1972.

33. PECK, S. Crown dimensions and the alignment or crowding of mandibular incisors. Am. J. Orthod. Dentofacial Orthop. St. Louis, v. 124, no. 4, p. 20A-2A, Oct. 2003.

34. PECK, S.; PECK, H. Crown dimensions and mandibular incisor alignment. Angle Orthod., Appleton, v. 42, no. 2, p. 148-153, Apr. 1972.

35. PUNEKY, P. J.; SADOWSKY, C.; BEGOLE, E. A. Tooth morphology and lower incisor alignment many years after orthodontic therapy. Am. J. Orthod., St. Louis, v. 86, no. 4, p. 299-305, Oct. 1984.

36. RHEE, S. H.; NAHM, D. S. Triangular-shaped incisor crowns and crowding. Am. J. Orthod. Dentofacial Orthop., St. Louis, v.118, no. 6, p. 624-648, Dec. 2000

37. RICHARDSON, M. E. Late lower arch crowding: the role of the transverse dimension. Am. J. Orthod. Dentofacial Orthop. St. Louis, v. 107, no. 6, p. 613-617, June 1995.

38. SADOWSKY, C.; SAKOLS, E. I. Long-term assessment of orthodontic relapse. Am. J. Orthod., St. Louis, v. 82, no. 6 , p. 456-463, Dec. 1982

39. SADOWSKY, C. et al. Long-term stability after orthodontic treatment: nonextraction with prolonged retention. Am. J. Orthod. Dentofacial Orthop., St. Louis, v. 106, no. 3, p. 243-249, Sept. 1994.

40. SANIN, C.; SAVARA, B. S. Factors that affect the alignment of the mandibular incisors: a longitudinal study. Am. J. Orthod., St. Louis, v. 64, no. 3, p. 248-257, Sept. 1973.

41. SHAH, A. A.; ELCOCK, C.; BROOK, A. H. Incisor crown shape and crowding. Am. J. Orthod. Dentofacial Orthop., St. Louis, v. 123, no. 5, p. 562-567, May 2003.

42. SHAPIRO, P. A. Mandibular dental arch form and dimension. Treatment and postretention changes. Am. J. Orthod. St. Louis, v. 66, no. 1, p. 58-70, July 1974.

43. SHERIDAN, J. J. Air-rotor stripping update. J. Clin. Orthod., Boulder, v. 21, no. 11, p. 781-788, Nov. 1987

44. SHIELDS, T. E.; LITTLE, R. M.; CHAPKO, M. K. Stability and relapse of mandibular anterior alignment: a cephalometric appraisal of first-premolar-extraction cases treated by traditional edgewise orthodontics. Am. J. Orthod., St. Louis, v. 87, no. 1 p. 27-38, Jan. 1985.

45. SINCLAIR, P. M.; LITTLE, R. M. Maturation of untreated normal occlusions. Am. J. Orthod., St. Louis, v. 83, no. 2, p. 114-123, Feb. 1983

46. SINCLAIR, P. M.; LITTLE, R. M. Dentofacial maturation of untreated normals. Am. J. Orthod., St. Louis, v. 88, no. 2, p. 146-156, Aug. 1985

47. SMITH, R. J.; DAVIDSON, W. M.; GIPE, D. P. Incisor shape and incisor crowding: a re-evaluation of the Peck and Peck ratio. Am. J. Orthod., St. Louis, v. 82, no. 3, p. 231-235, Sept. 1982.

48. VADEN, J. L.; HARRIS, E. F.; GARDNER, R. L. Relapse revisited. Am. J. Orthod. Dentofacial Orthop., St. Louis, v. 111, no. 5, p. 543-553, May 1997

49. VAN DER LINDEN, F. P. Theoretical and practical aspects of crowding in the human dentition. J. Am. Dent. Assoc., Chicago, v. 89, no. 1, p.139-153, July 1974.

50. YAMAGUTO, T. O. Determinação das medidas mesiodistais em indivíduos brasileiros leucodermas com oclusão normal. 2003. 108 f. Dissertação (Mestrado)-Universidade Metodista de São Paulo, São Bernardo do Campo, 2003.

\section{Endereço para correspondência}

Renata Cristina Faria Ribeiro de Castro

Departamento de Ortodontia

Alameda Dr. Octávio Pinheiro Brisolla n 9-75 / Vila Universitária

CEP: 17.043-900 - Bauru/SP

E-mail: drarenatacastro@superig.com.br 